\title{
Predicción de la satisfacción con la vida en practicantes de ejercicio físico saludable
}

\section{Prediction of life satisfaction in practicing healthy exercise}

\section{Previsão de satisfação com a vida em praticar exercício saudável}

\author{
Daniel Torregrosa, Noelia Belando, y Juan Antonio Moreno-Murcia
}

Universidad Miguel Hernández de Elche

\begin{abstract}
Resumen: El objetivo de este estudio fue analizar la relación del estilo controlador del técnico deportivo, la percepción de apoyo a la autonomía de los practicantes con respecto al técnico deportivo y los mediadores psicológicos con la satisfacción con la vida en practicantes de ejercicio físico saludable. Para ello se utilizó una muestra de 104 hombres practicantes de ejercicio físico no competitivo, con edades comprendidas entre los 18 y 70 años $(M=$ $28.38, D T=11.66)$ pertenecientes a seis centros de wellness espańoles. En el análisis de regresión lineal se observó que el estilo controlador predijo negativamente la satisfacción con la vida, mientras que el apoyo a la autonomía y los mediadores psicológicos predijeron positivamente la satisfacción con la vida. Los resultados se discuten en pro de la obtención de consecuencias positivas en los practicantes como una mayor satisfacción con sus vidas. Palabras clave: motivación autodeterminada; mediadores psicológicos; soporte de autonomía; actividad física.

Abstract: The aim of this study was to analyze the relationship of the controlling coach behavior, perceived autonomous support practitioners about the coach and the psychological mediators with the life satisfaction in healthy exercise practitioners. We used a sample of 104 noncompetitive male exercise practitioners aged between 18 and 70 years $(M=28.38$, SD $=11.66$ ) from six Spanish wellness centers. In linear regression analysis we
\end{abstract}

observed that the controlling coach behavior negatively effected life satisfaction, whereas autonomous support through psychological mediators positively effected life satisfaction. The results confirm our predicted effects on the life satisfaction of healthy exercise practitioners.

Key words: self-determination theory; psychological mediators; motivation; physical activity.

Resumo: O objetivo deste estudo foi analisar a relação entre o comportamento técnico de controle, apoio percebido sobre o treinador e os mediadores psicológicos Com a satisfação com a vida em praticantes de exercícios saudáveis. Utilizamos uma amostra de 104 praticantes de exercícios masculinos náo competitivos com idade entre 18 e 70 anos $(\mathrm{M}=28,38$, DP $=11,66)$ de seis centros de saúde espanhóis. Na análise de regressão linear observou-se que o comportamento técnico de controle efetuado negativamente satisfação com a vida, Considerando que o apoio autônoma através de mediadores psicológicos efetuada positivamente satisfação com a vida. Os resultados confirmam nossas efeitos previstos na satisfação com a vida de praticantes de exercícios saudáveis.

Palavras chave: teoria da autodeterminação; mediadores psicológicos; motivação; atividade física.

\section{Introducción}

Queda demostrado que la práctica de ejercicio físico a una intensidad entre moderada y vigorosa provoca mejoras en la salud (Warburton, 2009; Warburton, Nicol, y Bredin, 2006) a nivel fisiológico (Paffenbarger, Hyde, y Wing, 1990) y que contribuye al bienestar psicológico en general (Balaguer, Castillo, y Duda, 2008; Brown, 1990). En este sentido, el bienestar psicológico se caracteriza por un componente emocional y un componente cognitivo o también denominado satisfacción con la vida (Andrews y Withey, 1976). Siendo conscientes de que diversos estudios (Seager, 2012; Keyes, 2002) han constatado que un estado psico-social óptimo facilitaría la mejora del bienestar general de las personas, parece necesario

\footnotetext{
Dirección para correspondencia [Correspodence address]: Daniel Torregrosa García. Universidad Miguel Hernández de Elche. Departamento de Psicología de la Salud. Edificio Centro de Investigación del Deporte. Avenida de la Universidad, s/n. 03202 Elche (Alicante). E-mail: danitg88@gmail.com. Tel: +34620696062
}

seguir profundizando en el conocimiento de cuáles son las variables determinantes que podrían favorecer la aparición de una mayor satisfacción con la vida en practicantes de ejercicio físico saludable.

El modelo jerárquico de la motivación intrínseca y extrínseca (Vallerand, 1997, 2001) establece que pueden existir diversos factores o desencadenantes sociales que podrían influir sobre la conducta y motivación de los practicantes. Así, estudios realizados en el contexto de la competición deportiva manifiestan que la percepción del apoyo a la autonomía por parte del entrenador es un predictor positivo de la autonomía percibida por los practicantes (Balaguer et al., 2008). También se ha observado dicha relación en jóvenes deportistas de fútbol, críquet (Reinboth, Duda, y Ntoumanis, 2004) y en jóvenes gimnastas (Gagné, Ryan, y Bargmann, 2003). Asimismo, existen otros factores sociales como el entorno familiar, el grupo de iguales y los medios de comunicación que podrían determinar los estados emocionales del practicante 
(Nicholls, 1989). Sin embargo, los estudios de Balaguer et al. (2008) y Sarrazin, Vallerand, Guillet, Pelletier, y Cury (2002) indican que el factor social de percepción de apoyo a la autonomía (generado por el técnico deportivo) presenta el mayor poder de predicción de todos, pudiendo llevar al practicante a desarrollar una motivación más autodeterminada. De modo que, el apoyo a la autonomía por parte del técnico deportivo se caracteriza por la posibilidad de elección en las tareas, reduciendo así la necesidad de realizarlas por imposición del mismo y fomentando, por tanto, la iniciativa en la toma de decisiones del practicante (Deci y Ryan, 1985, 1991). Por el contrario, un técnico deportivo con un estilo controlador se caracteriza por la imposición de las tareas, de los pensamientos y metodologías pasivas donde el practicante no tiene opción a elegir y no es partícipe de su aprendizaje (Mageau y Vallerand, 2003). Estudios como el de Álvarez, Balaguer, Castillo, y Duda (2009) han relacionado positivamente el grado en que el practicante percibe el apoyo a la autonomía, por parte del técnico deportivo, con la satisfacción de las necesidades psicológicas básicas: autonomía (implicación en la toma de decisiones), competencia (sentir que se consiguen los objetivos planteados) y relación con los demás (calidad de la relaciones sociales). En este sentido, la teoría de la motivación autodeterminada (Deci y Ryan, 1985, 1991, 2000) establece que para que el practicante experimente mayor motivación intrínseca debe sentir satisfechas las tres necesidades psicológicas básicas. Dicha teoría, junto con diversos estudios (Pelletier, Fortier, Vallerand, y Briere, 2001; Pelletier, Fortier, Vallerand, Tuson, Brière, y Blais, 1995), han comprobado que la percepción de un clima controlador, está relacionado con una menor motivación intrínseca que la percepción de apoyo a la autonomía, lo que podría frustrar la satisfacción de los mediadores psicológicos. En esta misma línea, diferentes estudios desarrollados en el ámbito del deporte competitivo (Amorose y Anderson-Butcher, 2007; Conroy y Coatsworth, 2007), de la educación física (Hagger, Chatzisarantis, Hein, Pihu, Soós, y Karsai, 2007; Standage, Duda, y Notoumanis, 2005) y del ejercicio físico saludable (Edmunds, Ntoumanis, y Duda, 2006; Wilson y Rodgers, 2004) apoyan dicha relación. Además, varios estudios (Hagger y Chatzisarantis, 2007; Moraes, Corte-Real, Dias, y Fonseca, 2009) muestran una relación positiva entre la práctica físico-deportiva y una mayor satisfacción con la vida.

Así pues, el constructo satisfacción con la vida se entiende como la evaluación global que la persona hace sobre su vida, valorando los aspectos positivos y negativos y comparándolos con el modelo de vida establecido por el mismo (Pavot, Diener, Colvin, y Sandvik, 1991; Diener, 1984). Siguiendo a Andrews y Withey (1976) y Arthaud-Day, Rode, Mooney, y Nera (2005), la satisfacción con la vida es uno de los tres componentes de la percepción subjetiva de bienestar, siendo los otros dos las experiencias positivas y negativas. En esta línea, Zullig y White (2011) realizaron un estudio en el ámbito escolar que concluyó sugiriendo que la participación en actividades deportivas podría mejorar la satisfacción con la vida. En concordancia con lo anterior, Moreno-Murcia y Vera (2011) encontraron que la percepción del discente de una mayor autoestima y diversión predecían positivamente la satisfacción con la vida. En el caso de deportistas de competición, la mejora de la satisfacción con la vida podría estar determinada por los resultados deportivos, así como, por los estándares personales de mejora en dicho deporte (Gaudreau y Antl, 2008). No obstante, entre los trabajos encontrados aún no identificamos claras relaciones entre practicantes de ejercicio físico saludable y satisfacción con la vida. Al igual que los trabajos realizados por Huang y Humphrey (2012) en estadounidenses y Forrest y McHale (2009) en ingleses, en los que establecieron importantes diferencias entre la relación de la felicidad y la práctica de ejercicio físico en función de la población analizada.

Basándonos, por tanto, en los antecedentes presentados, el objetivo de la presente investigación consistió en comprobar el poder de predicción del apoyo a la autonomía, el estilo controlador del técnico deportivo y los mediadores psicológicos sobre la satisfacción con la vida en practicantes de ejercicio físico saludable. En analogía con los estudios revisados, la percepción de apoyo a la autonomía del practicante y los mediadores psicológicos predirían positivamente la satisfacción con la vida, al contrario que el estilo controlador, que lo haría negativamente.

Basándonos en los antecedentes presentado, con la intención de ampliar la literatura existente, en cuanto al estilo de intervención del técnico deportivo hacia metodologías más activas en la práctica de la actividad físico-deportiva saludable y debido a la escasez de estudios en dicho ámbito en relación a la satisfacción con la vida, el objetivo del estudio fue comprobar el poder de predicción de la percepción de apoyo a la autonomía, el estilo controlador del técnico deportivo y los mediadores psicológicos sobre la satisfacción con la vida de practicantes de fitness/salud. Tras el análisis de regresión lineal múltiple la hipótesis planteada fue confirmada.

\section{Método}

\section{Muestra}

La muestra estuvo compuesta por 104 hombres practicantes de ejercicio físico no competitivo, con edades comprendidas entre los 18 y 70 ańos $(M=28.38, D T=11.66)$ pertenecientes a seis centros españoles de wellness. Para seleccionar la muestra se llevó a cabo una selección de centros atendiendo a un muestreo aleatorio por conglomerados (Azorín y SánchezCrespo, 1986). 


\section{Medidas}

Estilo controlador. Se utilizó la escala Controlling Coach Behavior Scale (CCBS) de Bartholomew, Ntoumanis, y Thøgersen-Ntoumani (2010), validada al contexto español por Castillo et al. (2010). Mide los comportamientos controladores (imposiciones sobre las tareas) del técnico deportivo. Dicha escala consta de 15 ítems agrupados en cuatro factores: controlar el uso de las recompensas (e.g. "Me motiva prometiéndome una recompensa si lo hago bien”), condición negativa (e.g. "Se enfada conmigo si no me ejercito bien"), uso de la intimidación (e.g. "Me grita delante de los demás en la realización de los ejercicios") y excesivo control personal (e.g. "Espera que el ejercicio físico sea el centro de mi vida"). La frase previa es "Referente a la relación con mi monitor/a..." y las respuestas son recogidas en una escala tipo Likert de 1 (Totalmente en desacuerdo) a 7 (Totalmente de acuerdo). La consistencia interna fue de .88 para el control en el uso de la recompensa, .89 para la condición negativa, .92 para el uso de la intimidación y .92 para el excesivo control personal. Sin embargo, en el análisis de los datos se utilizó una única dimensión que engloba los cuatro factores descritos como "estilo controlador" con un alfa de Cronbach de .98.

Percepción de apoyo a la autonomía. Se utilizó la Escala de Apoyo a la Autonomía en Contextos de Ejercicio (PASSES) de Hagger et al. (2007) validada al contexto español por Moreno, Parra, y González-Cutre (2008), que mide la percepción de apoyo a la autonomía de los practicantes con respecto al técnico deportivo. Se compone de 11 ítems (e.g. "El monitor/a entiende por qué decido hacer ejercicio físico”). La oración previa es "En mis prácticas de ejercicio físico dirigido..." y las respuestas son recogidas en una escala tipo Likert de 1 (Totalmente en desacuerdo) a 7 (Totalmente de acuerdo). La consistencia interna fue de 97.

Mediadores psicológicos. Se utilizó la Escala de Medición de las Necesidades Psicológicas en el Ejercicio (PNSE) de Wilson, Rogers, Rodgers, y Wild (2006) validada al contexto español por Moreno-Murcia, Marzo, Martínez-Galindo, y Conte (2011). Consta de 18 ítems englobados en tres factores competencia (e.g. "Yo creo que puedo completar los ejercicios que son un reto personal"), autonomía, (e.g. "Siento que puedo hacer ejercicios a mi manera”) y relación con los demás (e.g. "Creo que me llevo bien con los que me relaciono cuando hacemos ejercicios juntos"). La oración previa es "En mis prácticas..." y las respuestas son recogidas en una escala tipo Likert que va de 1 (Falso) a 6 (Verdadero). La consistencia interna fue de .88 para la competencia, .88 para la autonomía, .85 para la relación con los demás. En el presente estudio se contemplaron los tres mediadores en un único factor que fue denominado "mediadores psicológicos" y que presentó una consistencia interna de .86 .

Satisfacción con la vida. Se utilizó la escala de satisfacción con la vida (ESDV-5) de Vallerand, Blais, Briére, y Pelletier (1989), validada al contexto español por Atienza, Pons, Balaguer, y García-Merita (2000) y Atienza, Balaguer, y GarcíaMerita (2003). Está formada por cinco ítems para valorar el factor satisfacción con la vida (e.g. "Estoy satisfecho con mi vida"). La sentencia previa es "Satisfacción con tu vida...." y las respuestas son recogidas en una escala tipo Likert que oscila de 1 (Totalmente en desacuerdo) a 7 (Totalmente de acuerdo). Se obtuvo un alfa de Cronbach de .92 .

\section{Procedimiento}

Se contactó con seis centros españoles de wellness a los que se les solicitó su colaboración y se les informó del objetivo de la investigación. La administración del cuestionario se realizó en el hall de cada centro en presencia del investigador principal, quien informó de los objetivos del estudio y resolvió las dudas que pudieron surgir. Fue necesario un tiempo aproximado de 15 minutos para su cumplimentación y se aseguró a los participantes la privacidad de los datos.

\section{Análisis de datos}

Se calcularon los estadísticos descriptivos de todas las variables objeto de estudio (medias y desviaciones típicas) y las correlaciones bivariadas. Para conocer la consistencia interna de cada dimensión se utilizó el coeficiente de alfa de Cronbach. Asimismo, se comprobó el poder predictivo del apoyo a la autonomía del técnico deportivo, el estilo controlador y los mediadores psicológicos sobre la satisfacción con la vida, a través de un análisis de regresión lineal múltiple por pasos. El tratamiento de los datos se realizó con el paquete estadístico SPSS 21.0.

\section{Resultados}

\section{Análisis descriptivo y correlaciones bivariadas}

El estilo controlador presentó una media de 3.09, la autonomía del monitor de 4.58, los mediadores psicológicos de 4.08 y la satisfacción con la vida de 5.13. Respecto al análisis de correlaciones bivariadas, el apoyo a la autonomía del técnico deportivo y los mediadores psicológicos correlacionaron positivamente con la satisfacción con la vida, mientras que el estilo controlador no correlacionó con ninguna variable (Tabla 1). 
Tabla 1. Estadísticos Descriptivo y Correlaciones de las Variables de Estudio

\begin{tabular}{lllllllll}
\hline Variables & $M$ & $D T$ & $\mathrm{R}$ & $\alpha$ & 1 & 2 & 3 & 4 \\
\hline 1. Estilo controlador & 3.09 & 1.45 & $1-7$ & .98 & - & .06 & .18 & -.08 \\
2. Autonomía monitor & 4.58 & 1.16 & $1-7$ & .97 & - & - & $.58^{* *}$ & $.49^{* *}$ \\
3. Mediadores psicológicos & 4.08 & .77 & $1-6$ & .86 & - & - & - & $.57^{* *}$ \\
4. Satisfacción con la vida & 5.13 & 1.17 & $1-7$ & .92 & - & - & - & - \\
\hline Nota: ${ }^{* *} p<.01 ; M=$ media; $D T=$ desviación típica; $\mathrm{R}=$ rango; $\alpha=$ alfa de Cronbach. \\
\hline
\end{tabular}

\section{Modelo de regresión lineal}

En el primer paso del análisis de regresión lineal el estilo controlador no explicó ningún porcentaje de la varianza. En el segundo paso, se introdujo el apoyo a la autonomía que predijo positivamente la satisfacción con la vida con un $23 \%$ de varianza explicada. En el tercer paso el estilo controlador predijo negativamente la satisfacción con la vida. Por su parte el apoyo a la autonomía del técnico deportivo y los mediadores psicológicos predijeron positivamente la satisfacción con la vida con un $37 \%$ de varianza explicada (Tabla 2).

Tabla 2. Análisis de Regresión Lineal de Predicción de la Satisfacción con la Vida a través del Estilo Controlador, Apoyo a la Autonomía del Técnico Deportivo y los Mediadores Psicológicos

\begin{tabular}{lllll}
\hline & $B$ & SEB & $b$ & $\mathrm{D} R^{2}$ \\
\hline Primer paso & 5.32 & .27 & & -.00 \\
Estilo controlador & -.06 & .08 & -.08 & \\
\hline Segundo paso & 3.12 & .46 & & $.23^{* *}$ \\
Estilo controlador & -.09 & .07 & -.11 & \\
Apoyo autonomía monitor & .50 & .009 & $.49^{* *}$ & \\
\hline Tercer paso & 1.64 & .52 & & $.37^{* *}$ \\
Estilo controlador & -.14 & .06 & $-.17^{*}$ & \\
Apoyo autonomía monitor & .23 & .10 & $.23^{*}$ & \\
Mediadores psicológicos & .70 & .15 & $.46^{* *}$ & \\
\hline Nota: ${ }^{*} p<.05 ;{ }^{* *} p<.01$. & & & & \\
\hline
\end{tabular}

\section{Discusión}

En concordancia con la teoría de la motivación autodeterminada, existe una relación positiva y significativa de la percepción de apoyo a la autonomía y la satisfacción de las necesidades psicológicas básicas (que como podemos observar en el análisis de regresión, actúa como mediador), con la satisfacción con la vida (percepción subjetiva de bienestar). Estudios publicados al respecto (Martín-Albo, Núñez, Domínguez, León, y Tomás, 2012; Balaguer, González, Fabra, Castillo, Mercé, y Duda, 2012; Castillo et al., 2010; Pelletier et al., 2001; Pelletier et al., 1995) concluyen que la percepción del practicante de un estilo controlador predice una menor motivación intrínseca. Aunque en dichos estudios no se relaciona directamente con la satisfacción con la vida, los resultados que se hallaron en este estudio sugieren que, además de lo ya descrito por dichos autores, la percepción de un estilo controlador podría reducir la satisfacción con la vida. En esta misma línea, estudios revisados en el contexto deportivo (Álvarez et al., 2009; Amorose y Anderson-Butcher, 2007; Conroy y coatsworth, 2007) muestran que la percepción por parte del practicante de apoyo a la autonomía predirá positivamente la satisfacción con la vida, estos resultados van en la misma línea que el presente trabajo, realizado en el contexto de ejercicio físico y salud. No obstante, Gaudreau y Antl (2008) describen que en el rendimiento deportivo, la satisfacción con la vida también puede depender de otras variables como los resultados deportivos o los estándares personales de mejora.

De modo que, tras considerar la evidencia del alto impacto del sedentarismo en la sociedad actual y bajo la necesidad de aumentar la adherencia al ejercicio físico, se podría concluir que la percepción de un apoyo a la autonomía por parte del practicante, así como, la satisfacción de sus necesidades psicológicas básicas, predicen positivamente la satisfacción con la vida, mientras que la percepción de un estilo controlador la predice negativamente. Esto podría ser determinante sobre la continuidad en un programa de entrenamiento físico-deportivo (Decy y Ryan, 1985, 1991,2000). Los resultados obtenidos en coherencia con los estudios considerados, podrían contribuir como guía para la planificación de programas de entrenamiento bajo metodologías que fomenten la autonomía de los practicantes (tareas abiertas, fomento de la iniciativa, elección de actividades, etc.) ya que parecen relacionarse con una consecución de las necesidades psicológicas básicas (Álvarez et al., 2009; Fortier, Sweet, O’Sullivan, y Williams, 2007) así como una mayor satisfacción con la vida (Philipe y Vallerand, 2008).

Respecto a las limitaciones del estudio, cabe destacar que la metodología utilizada ha sido de tipo correlacional. Esto nos ofrece una posibilidad futura de emplear una metodología experimental pues, aunque las variables manifiesten una relación matemática importante, no implica necesariamente que haya causa-efecto entre ellas (Quinata, 1989). Además, los datos no pueden ser extrapolados más allá de los rangos 
observados en la muestra del estudio. En este sentido, futuras investigaciones podrían profundizar en el análisis de la relación entre la percepción del apoyo a la autonomía y la satisfacción con la vida en poblaciones de diferentes edades a través de un programa activo de intervención bajo un seguimiento longitudinal.

\section{Referencias}

1. Álvarez, M. S., Balaguer, I., Castillo, I., y Duda, J. L. (2009). Coach autonomy support and quality of sport engagement in young soccer players. The Spanish Journal of Psychology, 12, 138-148.

2. Amorose, A. J., y Anderson-Butcher, D. (2007). Autonomy-supportive coaching and self-determined motivation in high school and college athletes: a test of self-determination theory. Psychology of Sport And Exercise, 8(5), 654-670.

3. Andrews, F. M., y Withey, S. B. (1976). Social indicators of well-being: America's perception of life quality. New York: Plenum.

4. Arthaud-Day, M. L., Rode, J. C., Mooney, C. H., y Near, J. P. (2005). The subjective well-being construct: a test of its convergent, discriminant, and factorial validity. Social Indicators Research, 74, 445-476.

5. Atienza, F. L., Balaguer, I., y Garcia-Merita, M. L. (2003). Satisfaction with life scale: analysis of factorial invariance across sexes. Personality and Individual Differences, 35(6), 1255-1260.

6. Atienza, F. L., Pons, D., Balaguer, I., y García-Merita, M. (2000). Propiedades psicométricas de la escala de satisfacción con la vida en adolescentes [Psychometric properties in the scale of life satisfaction in adolescents]. Psicothema, 12(2), 314-319.

7. Balaguer, I., Castillo, I., y Duda, J. L. (2008). Apoyo a la autonomía, satisfacción de las necesidades, motivación y bienestar en deportistas de competición: un análisis de la teoría de la autodeterminación [Autonomy support, needs satisfaction, motivation and well-being in competitive athletes: a test of the self-determination theory]. Revista de Psicología del Deporte, 17(1), 123-139.

8. Balaguer, I., González, L., Fabra, P., Castillo, I., Mercé, J., y Duda, J. L. (2012). Coaches interpersonal style, basic psychological needs and the well- and ill-being of young soccer players: a longitudinal analysis. Journal of Sport Science, 30(15), 1619-1629.

9. Bartholomew, K. J., Ntoumanis, N., y Thøgersen-Ntoumani, C. (2010). The controlling interpersonal style in a coaching context: development and initial validation of a psychometric scale. Journal of Sport \& Exercise Psychology, 32(2), 193-216.

10. Brown O. R. (1990). Exercise, fitness and mental health. En C. Bouchard, R. J. Shephard, T. Stephens, J. R. Sutton, y B. D. McPherson, (Ed.), Exercise, Fitness, and Health: a concensus of current knowledge (pp. 607-626). Illinois: Human Kinetics Books.

11. Castillo, I., Fabra, P., Marcos, D., González, L., Bartholomew, K. J., Fuentes, A., y Balaguer, I. (2010). El estilo controlador del técnico deportivo: Análisis de las propiedades psicométricas [Coach controlling interpersonal style: Analysis of psychometric properties]. En J. Cruz, (Ed.), Adaptation and validation of questionnaires in Sport Psychology. Symposium conducted at VII Iberoamerican Congress of Psychology, Oviedo, Asturias.

12. Conroy, D. E., y Coatsworth, J. (2007). Assessing autonomy-supportive coaching strategies in youth sport. Psychology of Sport And Exercise, 8(5), 671-684.

13. Deci, E. L., y Ryan, R. M. (1985). Intrinsic motivation and self-determination in human behavior. New York, USA: Plenum Press.

14. Deci, E. L., y Ryan, R. M. (1991). A motivational approach to self: integration in personality. En $R$. Dienstbier, (Ed.), Nebraska Symposium on Motivation: Perspectives on motivation (Vol. 38, pp. 237-288). Lincoln, USA: University of Nebraska Press.

15. Deci, E. L., y Ryan, R. M. (2000). The "what" and "why" of goal pursuits: Human needs and the self-determination of behavior. Psychologi- cal Inquiry, 11, 227-268.

16. Diener, E. (1984). Subjective well-being. Psychological Bulleting, 95, 542-575.

17. Edmunds, J., Ntoumanis, N., y Duda, J. L. (2006). A Test of Self-Determination Theory in the Exercise Domain. Journal Of Applied Social Psychology, 36(9), 2240-2265.

18. Forrest, D., y McHale, I. (2009). Public policy, sport and happiness: An empirical study. Working paper, Salford University.

19. Fortier, M. S., Sweet, S. N., O'Sullivan, T. L., y Williams, G. C. ( 2007). A self-determination process model of physical activity adoption in the context of a randomized controlled trial. Psychology of Sport and Exercise, $8,741-757$.

20. Gagné, M., Ryan, R. M., y Bargmann, K. (2003). Autonomy support and need satisfaction in the motivation and well-being of gymnasts. Journal of Applied Sport Psychology, 15, 372-390.

21. Gaudreau, P., y Antl, S. (2008). Athletes' broad dimensions of dispositional perfectionism: examining changes in life satisfaction and the mediating role of sport-related motivation and coping. Journal of Sport \& Exercise Psychology, 30, 356-382.

22. Hagger, M. S., Chatzisarantis, N. D., Hein, V., Pihu, M., Soós, I., y Karsai, I. (2007). The perceived autonomy support scale for exercise settings (PASSES): development, validity, and cross-cultural invariance in young people. Psychology of Sport and Exercise, 8(5), 632-653.

23. Huang, H., y Humphreys, B. R. (2012). Sport participation and happiness: evidence from US microdata. Journal of Economic Psychology, 33, 776-793.

24. Keyes, C. M. (2002). The mental health continuum: From languishing to flourishing in life. Journal Of Health And Social Behavior, 43(2), 207222.

25. Mageau, G. A., y Vallerand, R. J. (2003). The coach-athlete relationship: a motivational model. Journal of Sports Sciences, 21, 883-904.

26. Martín-Albo, J., Núñez, J. L., Domínguez, E., León, J., Tomás, J. M., (2012). Relationships between intrinsic motivation, physical self-concept and satisfaction with life: a longitudinal study. Journal of Sport Sciences, 30(4), 337-347.

27. Moraes, M., Corte-Real, N., Dias, C., y Fonseca, A. M. (2009). Satisfaçao com a vida, exercício físico e consumo de tabaco em adolescentes de diferentes áreas geográficas de Portugal. Revista Brasileña de Ciencias del Deporte, 30(2), 137-149.

28. Moreno, J. A., Parra, N., y González-Cutre, D. (2008). Influencia del apoyo a la autonomía, las metas sociales y la relación con los demás sobre la desmotivación en educación física [Influence of autonomy support, social goals and relatedness on amotivation in physical education classes]. Psicothema, 20(4), 636-641.

29. Moreno-Murcia, J. A., Marzo, J. C., Martínez-Galindo, C., y Conte, L. (2011). Validación de la escala de "Satisfacción de las Necesidades Psicológicas Básicas" y del cuestionario de la "Regulación Conductual en el Deporte" al contexto español [Validation of Psychological Need Satisfaction in Exercise Scale and the Behavioural Regulation in Sport Questionnaire to the Spanish context]. RICYDE. Revista Internacional de Ciencias del Deporte, 26, 355-369.

30. Moreno-Murcia, J. A., y Vera, J. A. (2011). Modelo causal de la satisfacción con la vida en adolescentes de educación física [Model causal of the satisfaction with the life in adolescent students of physical education]. Revista de Psicodidáctica, 16(2), 367-380. 
31. Moreno, J. A., González-Cutre, D., Chillón, M., y Parra, N. (2008). Adaptación a la educación física de la escala de las necesidades psicológicas básicas en el ejercicio [Physical education Adjustment Scale basic psychological needs in exercise]. Revista Mexicana de Psicología, 25(2), 295-303.

32. Nicholls, J. G. (1989). The competitive ethos and democratic education. Cambridge, MASS: Harvard University Press.

33. Paffenbarger R. S. Jr., Hyde R. T., y Wing A. L. (1990) Physical activity and physical fitness as determinants of health and longevity. En C. Bouchard, R. J. Shephard, T. Stephens, J. R. Sutton, y B. D. McPherson, (Ed.), Exercise, Fitness, and Health: A Concensus of Current Knowledge (pp. 33-48). Illinois: Human Kinetics Books.

34. Pavot, W., Diener, E., Colvin, C. R., y Sandvik, E. (1991). Further validation of the satisfaction with life scale: evidence for the cross-method convergence of well-being measures. Journal of Personality Assessment, 57, 149-161.

35. Pelletier, L. G., Fortier, M. S., Vallerand, R. J., y Brière, N. M. (2001). Perceived autonomy support, levels of self-determination, and persistence for an activity: a longitudinal investigation. Motivation and Emotion, 25, 279-306.

36. Pelletier, L. G., Fortier, M. S., Vallerand, R. J., Tuson, K. M., Brière, N. M., y Blais, M. R. (1995). Toward a new measure of intrinsic motivation, extrinsic motivation, and amotivation in sports: the sport motivation scale (SMS). Journal of Sport and Exercise Psychology, 17, 35-53.

37. Philippe, F. L., y Vallerand, R. J. (2008). Actual environments do affect motivation and psychological adjustment: a test of self-determination theory in a natural setting. Motivation and Emotion, 32(2), 81-89.

38. Quintana, C. (1989). Elementos de inferencia estadistica [Elements of statistical inference]. Costa Rica: Editorial de la Universidad de Costa Rica.

39. Reinboth, M., Duda, J. L., y Ntoumanis, N. (2004). Dimensions of coaching behavior, need satisfacion, and the psychological and physical welfare of young athletes. Motivation and Emotion, 28(3), 297-313.

40. Sarrazin, P., Vallerand, R., Guillet, E., Pelletier, L., y Cury, F. (2002).
Motivation and dropout in female handballers: a 21-month prospective study. European Journal of Social Psychology, 32, 395-418.

41. Seager, M. (2012). Towards a public health psychology. Journal of Public Mental Health, 11(3), 153-156.

42. Standage, M., Duda, J. L., y Ntoumanis, N. (2005). A test of selfdetermination theory in school physical education. British Journal of Educational Psychology, 75(3), 411-433.

43. Vallerand, R. J. (1997). Toward a hierarchical model of intrinsic and extrinsic motivation. En M. P. Zanna (Ed.), Advances in experimental social psychology (pp. 271-360). New York: Academic Press.

44. Vallerand, R. J. (2001). A hierarchical model of intrinsic and extrinsic motivation in sport and exercise. En G. C. Roberts (Ed.), Advances in motivation in sport and exercise (pp. 263-319). Champaign, IL: Human Kinetics.

45. Vallerand, R. J., Blais, M. R., Brière, N. M., y Pelletier, L. G. (1989). Construction et validation de l'Échelle de Motivation en Éducation (EME). Canadian Journal of Behavioral Sciences, 21(3), 323-349.

46. Warburton, D. E. R. (2009). The physical activity and exercise continuum. En C. Bouchard, y P. T. Katzmarzyk, (Ed.), Advances in physical activity and obesity (pp. 21-30). Champaign IL: Human Kinetics Publishing.

47. Warburton, D. E. R., Nicol, C., y Bredin, S. S. (2006). Health benefits of physical activity: the evidence. Canadian Medical Association Journal, 174(6), 801-809.

48. Wilson, P. M., y Rodgers, W. M. (2004). The relationship between perceived autonomy support, exercise regulations and behavioral intentions in women. Psychology Of Sport And Exercise, 5(3), 229-242.

49. Wilson, P. M., Rogers, W., Rodgers, W. M., y Wild, T. (2006). The psychological need satisfaction in exercise scale. Journal of Sport \& Exercise Psychology, 28(3), 231-251.

50. Zullig, K. J., y White, R. J. (2011). Physical activity, life satisfaction, and self-rated health of middle school students. Applied Research Quality Life, 6, 277-289. 